The Astrophysical Journal, 672:1196-1201, 2008 January 10

(C) 2008. The American Astronomical Society. All rights reserved. Printed in U.S.A.

\title{
A SURVEY FOR MASSIVE GIANT PLANETS IN DEBRIS DISKS WITH EVACUATED INNER CAVITIES ${ }^{1}$
}

\author{
D. $\mathrm{APAI}^{2}$ \\ Steward Observatory, University of Arizona, Tucson, AZ 85721; apai@as.arizona.edu \\ M. JANSON \\ Max Planck Institute for Astronomy, Königstuhl 17, Heidelberg, D-69117, Germany
}

A. Moro-Martín

Department of Astrophysical Sciences, Peyton Hall, Ivy Lane, Princeton University, Princeton, NJ 08544

M. R. MEYER ${ }^{2}$

Steward Observatory, University of Arizona, Tucson, AZ 85721

E. E. MamajeK

Harvard-Smithsonian Center for Astrophysics, 60 Garden Street, MS-42, Cambridge, MA 02138

E. MASCIADRI

INAF-Astrophysical Observatory Arcetri, Italy

Th. Henning

Max Planck Institute for Astronomy, Königstuhl 17, Heidelberg, D-69117, Germany

I. PASCUCCI AND J. S. KIM

Steward Observatory, University of Arizona, Tucson, AZ 85721

L. A. Hillenbrand

California Institute of Technology, Pasadena, CA 91125

M. KASPER

European Southern Observatory, Karl-Schwarzschild-Strasse. 2, D-85748 Garching, Germany

AND

B. BILLER

Steward Observatory, University of Arizona, Tucson, AZ 85721

Received 2007 July 6; accepted 2007 September 24

\begin{abstract}
The commonality of collisionally replenished debris around main-sequence stars suggests that minor bodies are frequent around Sun-like stars. Whether or not debris disks in general are accompanied by planets is yet unknown, but debris disks with large inner cavities - perhaps dynamically cleared — are considered to be prime candidates for hosting large-separation massive giant planets. We present here a high-contrast VLT/NACO angular differential imaging survey for eight such cold debris disks. We investigated the presence of massive giant planets in the range of orbital radii where the inner edge of the dust debris is expected. Our observations are sensitive to planets and brown dwarfs with masses >3-7 Jupiter mass, depending on the age and distance of the target star. Our observations did not identify any planet candidates. We compare the derived planet mass upper limits to the minimum planet mass required to dynamically clear the inner disks. While we cannot exclude that single giant planets are responsible for clearing out the inner debris disks, our observations constrain the parameter space available for such planets. The nondetection of massive planets in these evacuated debris disks further reinforces the notion that the giant planet population is confined to the inner disk $(<15 \mathrm{AU})$.
\end{abstract}

Subject headings: circumstellar matter — planetary systems — stars: individual (HD 105, HD 377, HD 107146, HD 202917, HD 209253, HD 35850, HD 70573, HD 25457)

\section{INTRODUCTION}

Collisionally replenished debris dust surrounds about $10 \%-$ $20 \%$ of the main-sequence Sun-like stars (e.g., Meyer et al. 2007). Such widespread evidence for minor body collisions demonstrates that planetesimals orbit most stars. It is natural to ask whether or not rocky and giant planets are also present in these systems. No convincing correlation could yet be found between close-in exo-

\footnotetext{
1 Based on observations collected at the European Southern Observatory at Paranal, Chile (ESO programs P078.C-0412(A) and P077.C-0391(A)).

2 Laplace Team, NASA Astrobiology Institute.
}

planets and the presence of debris (e.g., Moro-Martín et al. 2007a: but see Beichman et al. 2005). However, the presence of massive giant planets has been often invoked to account for the observed azimuthal or radial asymmetries at large radii in many debris disks (e.g., Greaves et al. 2005; Wilner et al. 2002). While theory offers several alternative mechanisms (e.g., Takeuchi \& Artymowicz 2001; Wyatt 2005), dynamical clearing of dust parent bodies by giant planets remains a feasible and exciting theoretical possibility (e.g., Moro-Martín \& Malhotra 2005; Quillen 2006; Levison et al. 2007; Morbidelli et al. 2007).

Examples for such possibly dynamically cleared disks include two recently identified disks around the young Sun-like stars 
TABLE 1

Target Parameters

\begin{tabular}{|c|c|c|c|c|c|c|c|c|}
\hline \multirow[b]{2}{*}{ TARGET } & \multirow{2}{*}{$\begin{array}{c}\text { R.A. } \\
\text { (J2000.0) }\end{array}$} & \multirow{2}{*}{$\begin{array}{c}\text { Decl. } \\
(\mathrm{J} 2000.0)\end{array}$} & \multirow[b]{2}{*}{$V$ MAGNITUDE $^{\mathrm{b}}$} & \multirow{2}{*}{$\begin{array}{l}\text { Distance }^{\mathrm{b}} \\
(\mathrm{pc})\end{array}$} & \multirow[b]{2}{*}{ Spectral Type } & \multicolumn{3}{|c|}{$\operatorname{Ages}(\mathrm{Myr})^{\mathrm{a}}$} \\
\hline & & & & & & $t_{\min }$ & $t_{\text {prob }}$ & $t_{\max }$ \\
\hline HD 105 & 000552.6 & -414511 & 7.51 & 40 & G0 V & 27 & 30 & 225 \\
\hline HD 377 ................................... & 000825.7 & +063701 & 7.59 & 40 & $\mathrm{G} 2 \mathrm{~V}$ & 25 & 90 & 220 \\
\hline 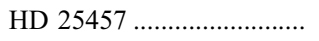 & 040236.8 & -001608 & 5.38 & 19 & F5 V & 50 & 75 & 170 \\
\hline 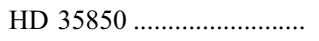 & 052704.8 & -115403 & 6.30 & 27 & $\mathrm{~F} 7 / 8 \mathrm{~V}$ & 12 & 12 & 100 \\
\hline 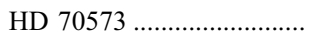 & 082250.0 & +015134 & 8.69 & 70 & $\mathrm{G} 1 / 2 \mathrm{~V}$ & 30 & 60 & 125 \\
\hline HD 107146 & 121906.5 & +163254 & 7.04 & 29 & G2 V & 80 & $\ldots$ & 200 \\
\hline 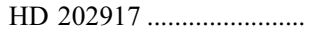 & 212050.0 & $\begin{array}{llll}-53 & 02 & 03\end{array}$ & 8.65 & 46 & G5 V & 30 & 30 & 100 \\
\hline HD 209253 & 220233.0 & -320802 & 6.63 & 30 & $\mathrm{~F} 6 / 7 \mathrm{~V}$ & 200 & 500 & 1600 \\
\hline
\end{tabular}

NoTE.-Units of right ascension are hours, minutes, and seconds, and units of declination are degrees, arcminutes, and arcseconds.

${ }^{a}$ The age estimates are discussed in the text.

${ }^{\mathrm{b}}$ All magnitudes and distances from the Hipparcos catalog, except for the distance of HD 70573, which is a main-sequence distance.

HD 105 (Meyer et al. 2004) and HD 107146 (Williams et al. 2004). Both disks were found to exhibit strong excess emission at wavelengths longer than $30 \mu \mathrm{m}$, while displaying no measurable excesses shortward of $20 \mu \mathrm{m}$. The detailed analysis of the spectral energy distribution of HD 105 suggests that it is consistent with a narrow dust ring $(<4 \mathrm{AU})$ with an inner radius of $\sim 42 \mathrm{AU}$, if the dust grains emit like blackbodies (Meyer et al. 2004). Using a similar model Williams et al. (2004) showed that the excess emission from HD 107146 is consistent with arising from cold dust $(T=51 \mathrm{~K})$ emitting as a single-temperature blackbody. The lack of measurable infrared excess shortward of $25 \mu \mathrm{m}$ illustrates that the inner disk regions are well cleared of dust: for HD 107146 there is at most 140 times less warm $\operatorname{dust}(T=100 \mathrm{~K})$ than cold dust ( $T=51 \mathrm{~K}$, Williams et al. 2004). The findings of the spectral energy distribution model for HD 107146 have been confirmed by direct imaging with the Hubble Space Telescope, that strengthen the case for a large featureless dust ring outside of an evacuated inner cavity (Ardila et al. 2004).

Recent high-contrast imaging surveys have hinted on the general scarcity of giant planets at such large separations (e.g., Masciadri et al. 2005; Kasper et al. 2007; Biller et al. 2007; Lafreniere et al. 2007). Quantitative statistical analysis of the nondetections demonstrates that - at a $90 \%$ confidence levelthe giant planet population cannot extend beyond $30 \mathrm{AU}$ if it follows a $r^{0.2}$ radial distribution, consistent with the radial velocity surveys. The statistical analysis suggests an outer cut-off for the giant planet population at $<15 \mathrm{AU}$ (Kasper et al. 2007). If so, dynamically cleared cold debris disks may be the signposts for rare large-separation giant planets, ideally suited for direct imaging studies.

In this paper we report on a VLT/NACO high-contrast imaging survey for large-separation giant planets around HD 105, HD 107146, and six other similar disks. In the following we review the target stars and disks and the observations, following with a comparison of our nondetections to lower planet mass limits set by dynamical clearing simulations.

\subsection{Targets}

Our targets were selected from the sample of 328 Sun-like stars $\left(0.7-2.2 M_{\odot}\right)$ targeted in the Formation and Evolution of Planetary Systems Spitzer Space Telescope Legacy program (FEPS; Meyer et al. 2006). From this sample we identified eight southern stars that (1) display strong infrared excess emission at long wavelengths ( $\lambda>20 \mu \mathrm{m}) ;(2)$ have no measurable excess emission at shorter wavelengths; and, (3) are young and close enough to permit the detection of planetary-mass objects within the inner radius of the cold debris. Table 1 gives an overview of the key parameters of the target stars. The typical lower mass limit for the debris in the systems is $10^{-4}$ to $10^{-5} M_{\oplus}$, making these disks massive analogs of our Kuiper Belt (Meyer et al. 2004; Kim et al. 2005; Hillenbrand et al. 2007). The disks of HD 105 and HD $107146-$ included in our sample - have inner evacuated regions with an estimated radii of $\sim 40$ and $\sim 31 \mathrm{AU}$ (Meyer et al. 2004; Williams et al. 2004). The other six disks exhibit spectral energy distributions similar to HD 105 and HD 107146. Based on the similarity of the excess emissions and the almost identical spectral types, all eight disks are expected to have cleared-out inner disks of similar size. The only possible exception in this sample is HD 202917, for which the recalibration of the IRAC fluxes after our VLT observations revealed a faint, but likely real infrared excess even at wavelengths shortward of $10 \mu \mathrm{m}$, suggesting that this inner disk may harbor small, but nonnegligible amounts of warm dust.

In the following we discuss briefly the results of the age determination for these sources as this has direct impact on the sensitivity of our observations to giant planets. A more detailed discussion of the ages of the whole FEPS sample will be presented in L. Hillenbrand et al. (2007). We briefly summarize the upper and lower age estimates $\left(t_{\min }\right.$ and $\left.t_{\max }\right)$ for each star, along with the most likely age $t_{\text {prob }}$, where available. HD 105 has already reached the main sequence $\left(t_{\min }=27 \mathrm{Myr}\right)$ and its chromospheric activity suggests a $t_{\max }$ of $225 \mathrm{Myr}$ (Hillenbrand et al. 2007). It is very likely a member of the Tuc-Hor moving group (Mamajek et al. 2004), and its $t_{\text {prob }}$ is $30 \mathrm{Myr}$ (Hollenbach et al. 2005). HD 377 is also a main-sequence $\operatorname{star}\left(t_{\min }>25 \mathrm{Myr}\right)$, and the chromospheric activity suggests that $t_{\max }=220 \mathrm{Myr}$. The median of four other age indicators sets $t_{\mathrm{prob}}=90 \mathrm{Myr}$. For HD 107146 we adopt the age range of $80-200$ Myr. HD 202917 is a likely member of the Tuc-Hor moving group $\left(t_{\min }=t_{\text {prob }}=30 \mathrm{Myr}\right)$, and its upper age limit is set by its Li abundance, higher than that of the Pleiades $\left(t_{\max }<100 \mathrm{Myr}\right)$. HD 35850 is suggested to be a $\beta$ Pic Moving Group member $\left(t_{\min }=12 \mathrm{Myr}\right.$; Song et al. 2003), and its observed rotation rate sets a reliable upper age limit of $t_{\max }=100 \mathrm{Myr}$ (Hillenbrand et al. 2007; see Barnes 2007). HD 70573 is among the few stars that are known to harbor both a debris disk and a giant planet. Setiawan et al. (2007) found an $m_{2} \sin i=6.1 M_{\mathrm{J}}$ possible planet on a $1.76 \mathrm{AU}$ orbit. A combination of different age indicators suggest a $t_{\min }=30 \mathrm{Myr}$ for HD 70573 and a $t_{\text {prob }}=60 \mathrm{Myr}$; Setiawan et al. (2007) quotes $t_{\max }=125$ Myr. Based on Li abundance and chromospheric activity, position on the color-magnitude diagram, and the analysis of its space motions, E. E. Mamajek et al. (in preparation) estimate that HD 209253 has $t_{\min }=200 \mathrm{Myr}$ and $t_{\max }<1.6 \mathrm{Gyr}$ with 
TABLE 2

Log OF THE OBSERVATIONS

\begin{tabular}{|c|c|c|c|c|c|c|}
\hline Target & UT Dates & $\begin{array}{c}\text { NDIT } \times \text { DIT }^{a} \\
(\mathrm{~s})\end{array}$ & Frames per Angle & $\begin{array}{l}\text { Angles } \\
\text { (deg) }\end{array}$ & $\begin{array}{l}\text { On Source } \\
\text { (minute) }\end{array}$ & $\begin{array}{c}\text { Strehl } \\
(\%)\end{array}$ \\
\hline 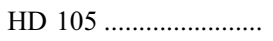 & 2006 Jul 20, Aug 18, and Aug 21 & $1 \times 4$ & 144 & 0,33 & 19 & 43 \\
\hline 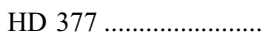 & 2006 Aug 27 & $1 \times 4$ & 144 & 0,33 & 19 & 37 \\
\hline 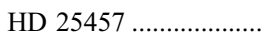 & 2006 Aug 12 and 13 & $1 \times 4$ & 144 & 0,33 & 19 & 46 \\
\hline 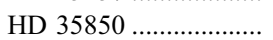 & 2006 Aug 13 & $1 \times 4$ & 144 & 0,33 & 19 & 40 \\
\hline 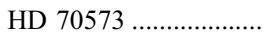 & 2007 Mar 2 & $21 \times 5$ & 16 & 0,33 & 56 & 58 \\
\hline HD $107146 \ldots \ldots \ldots \ldots \ldots . .$. & 2006 Apr 26 and May 26 & $1 \times 4$ & 95 & 0,33 & 13 & 51 \\
\hline HD $202917 \ldots \ldots \ldots \ldots \ldots . . .$. & 2006 June 23 & $1 \times 12$ & 32 & 0,33 & 13 & 45 \\
\hline HD $209253 \ldots \ldots \ldots \ldots \ldots . . .$. & 2006 July 9,16 , and 23 & $1 \times 4$ & 144 & 0,33 & 19 & 49 \\
\hline
\end{tabular}

${ }^{\mathrm{a}}$ DIT $=$ detector integration time; NDIT $=$ number of integration averaged on-chip.

$t_{\text {prob }}=500$ Myr. HD 25457 is a member of the AB Dor moving group giving a very strong lower age limit $\left(t_{\min }=50 \mathrm{Myr}\right.$ : Zuckerman et al. 2004). Luhman et al. (2005) derive an age of 75-125 Myr (we adopt $t_{\text {prob }}=75 \mathrm{Myr}$ ), while the upper age limit is set by the chromospheric activity $\left(t_{\max }=170 \mathrm{Myr}\right)$.

\section{OBSERVATIONS AND DATA REDUCTION}

Our eight targets were observed with ESO's Very Large Telescope using the NACO adaptive optics system (Lenzen et al. 2003; Rousset et al. 2003). The observations were carried out in service mode in late 2006 and early 2007 (see Table 2 for the log of the observations). The weather conditions were excellent with typical visual seeing of $0.8^{\prime \prime}$ and clear skies.

We used the spectral differential imaging mode (SDI) of NACO in order to enhance the contrast for any methane-rich cold $(T<$ $1200 \mathrm{~K}$ ) companion (e.g., Lenzen et al. 2004). The SDI mode uses two Wollaston prisms to split the incoming light rays into four beams of nearly identical light path. These rays pass through four narrowband filters, two of which are identical. The three different filters $\left(f_{1}, f_{2}\right.$ and $f_{3}$ corresponding to $1.575,1.600$, and $1.625 \mu \mathrm{m}$ ) probe the $1.62 \mu \mathrm{m}$ methane feature and the adjacent continuum. Because the SDI mode uses the $1024 \times 1024$ pixel S13 camera of NACO with a $13^{\prime \prime} \times 13^{\prime \prime}$ field of view, the simultaneous acquisition of four images in this field reduces the effective field of view to about $3^{\prime \prime} \times 3^{\prime \prime}$.

The achieved contrast, however, is not as good as predicted, probably due to the combined effect of readout noise and a slightly lower Strehl ratio. With the reduced contrast our observations were sensitive only to planets beyond the $1-3 M_{\mathrm{J}}$ planet mass range. These planets - at the young ages of out targets - are too hot to display the $1.62 \mu \mathrm{m}$ methane feature (Burrows et al. 2003). The lack of the methane feature results in almost identical planet fluxes in the $f_{1}, f_{2}$, and $f_{3}$ filters, rendering the SDI technique inefficient.

Instead, we opted to reduce the data taken in the $f_{1}$ filter $\left(\lambda_{c}=\right.$ $1.575 \mu \mathrm{m}, \delta \lambda=0.025 \mu \mathrm{m})$ in the angular differential imaging mode, i.e., without applying the spectral differential imaging step (e.g., Mueller \& Weigelt 1987; Marois et al. 2006; Kasper et al. 2007). The data reduction was performed with a dedicated pipeline, as described in detail in Kellner (2005) and Janson et al. (2007). The frames taken at a given rotator angle were averaged and the collapsed frame corresponding to one angle was subtracted from the other. This procedure cancels out residual static or quasistatic features from the instrument, whereas any companion will remain as a combination of a positive and negative point source. The intensity of the residuals (at a certain separation from the primary) is characterized by taking the standard deviation in a $9 \times 9$ pixel square $\left[0.11^{\prime \prime} \times 0.11^{\prime \prime} \approx(2.7 \lambda / D)^{2}\right]$ centered on that separation, at 180 evenly sampled angles, and taking the median of the results. This is repeated for all separations to create a radial profile of the error distribution in the image. When combined with the brightness of the primary, this yields the achieved contrast as a function of separation in the final image (see, Janson et al. 2007 for more details). Previous artificial planet tests on identical data sets processed with the reduction pipeline used here showed that $3 \sigma$ bright sources would have been reliably identified as candidate planets (Janson et al. 2007). Lacking any such detection, we used $3 \sigma$ fluxes as upper limits on the brightness of any companions to the target stars. In order to convert the achieved contrast in $f_{1}$ to the more commonly used $H$ band, we derived a conversion factor by comparing the flux densities in the two filters in a simulated spectrum of a giant planet in the age and mass range probed by our observations (Burrows et al. 2003).

\section{RESULTS}

The NACO/ADI observations acquired high-contrast, highresolution images of the eight target stars and their immediate environment (typically between 10 and $70 \mathrm{AU}$ ). In spite of the sensitive observations we could not identify giant planet candidates or any other point sources in the images.

Our data analysis allows us to set firm upper limits to the brightness of the sources that would have been identified as a candidate. We use the age estimates in Table 1 and planetary evolution models (Baraffe et al. 2003) to convert the achieved sensitivities to planet masses. These limits are shown in Figure 1 as a function of separation from the target stars for the lower and upper age limits of our stars.

Using the high-contrast observations we can study the probable range of radii in these disks at which the dust debris and the parent body planetesimals reside. Our measurements exclude the presence of any brown dwarf companions for virtually all our targets at these radii. For HD 209253, the oldest star in our sample, the images exclude companions down to the brown dwarf/giant planet mass boundary $\left(13 M_{\mathrm{J}}\right)$ at radii $20 \mathrm{AU}$ or greater. For the youngest source HD 35850 (12-100 Myr) our observations exclude any giant planet companions down to 3-4 $M_{\mathrm{J}}$ between 25 and $45 \mathrm{AU}$, if the star belongs to the $\beta$ Pic moving group as suggested by Song et al. (2003). For the other six sources our observations are typically sensitive to $\sim 6 M_{\mathrm{J}}$ at orbital radii $>30-40 \mathrm{AU}$.

Note that the dominant uncertainty of the upper limits stems from the difficulty of stellar age determination and from the poorly constrained initial conditions for giant planet evolution models. In particular, if shocks lead to efficient energy dissipation during the accretion phase, giant planets may start with much lower 


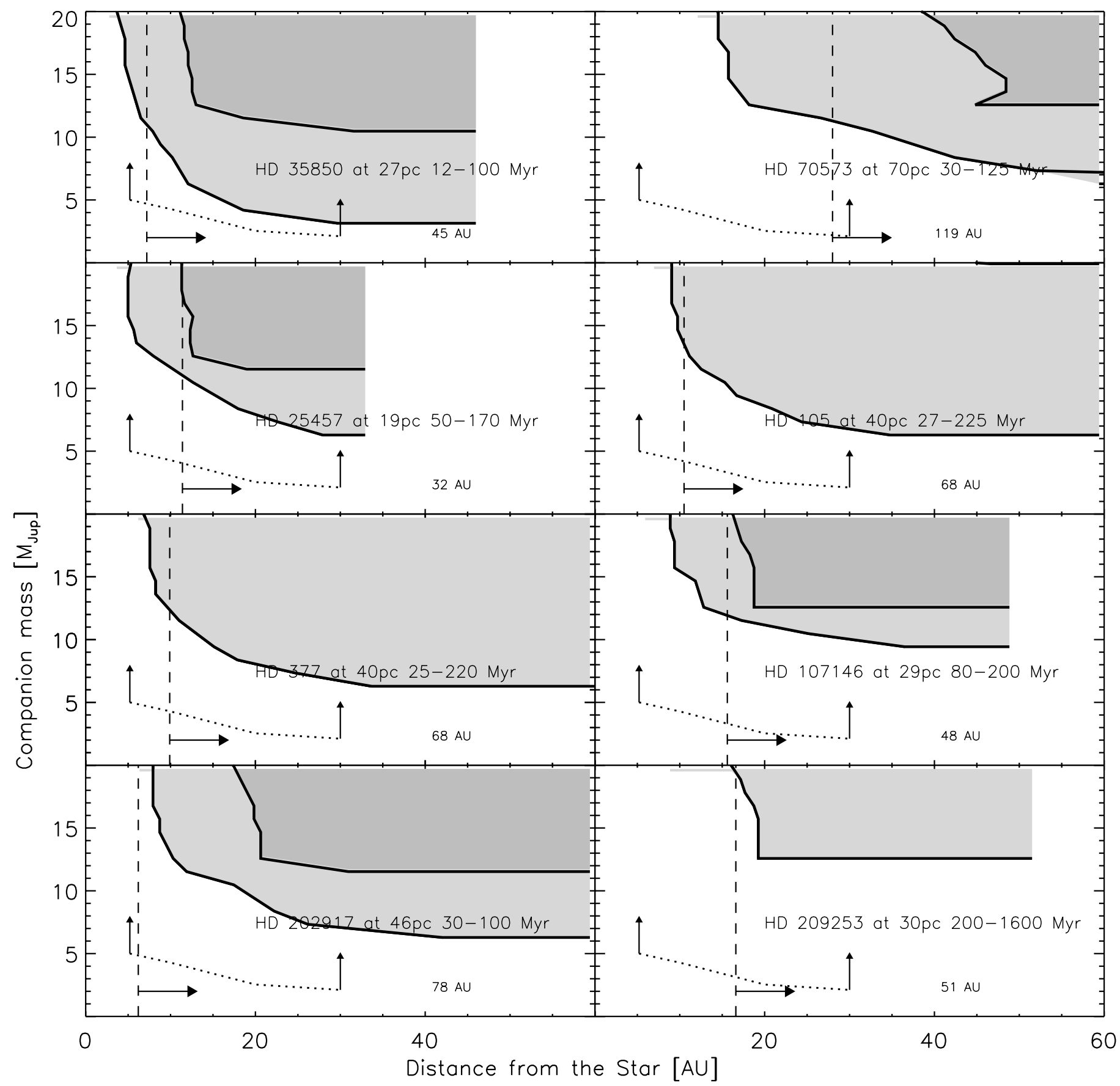

FIG. 1.-Discovery space of the NACO observations (shaded area), the minimum predicted planet masses required for a single planet to scatter out $>90 \%$ of the planetesimals at a given radius (dotted lines), and the lower limits for the disk inner radius (dashed lines; Hillenbrand et al. 2007). The presence of planets within the shaded parameter range is excluded by our observations. The upper and lower shaded sensitivity curves mark the limits for a possible younger and older stellar age. For each figure the outer radius of the field of view is given in astronomical units in the lower right-hand corner. Note, that the reversing sensitivity curve for HD 70573 is due to the reversal of the planet mass-luminosity curve in the corresponding evolutionary phase (Baraffe et al. 2003).

luminosities (e.g., Marley et al. 2007) than assumed by the hotstart models (e.g., Baraffe et al. 2003; Burrows et al. 2003).

\section{DISCUSSION: INSIDE-EVACUATED DEBRIS DISKS WITHOUT MASSIVE GIANT PLANETS?}

If single planets are responsible for clearing out the inner disks in the observed systems, they will be located very close to the inner edge of the debris. Although the available data does not allow the direct measurement of the inner disk radii, simple blackbody fits to the spectral energy distributions provide reliable lower limits. We adopt these limits from Hillenbrand et al. (2007) and note that they range from 6.2 to 28 AU. Figure 1 shows the limits for the individual sources (dashed lines). We stress that these are lower limits - the real inner disk radii are probably somewhat larger.

Given the range of detectable planet masses in Figure 1, we assess whether dynamical clearing by a less massive and therefore undetectable planet could still be a feasible mechanism to explain the lack of measurable quantities of warm dust, or on the contrary, if the dynamical clearing scenario may be rejected. Because our disks are devoid of gas (Pascucci et al. 2006), we use the dynamical models by Moro-Martín \& Malhotra (2005) to investigate 
the effect of a giant planet on the dust population. These models investigate the efficiency of dust particle ejection by gravitational scattering as a function of planet mass and planet location. In these models the dust particles are released from an outer belt of planetesimals and drift inward toward the central star under the effect of Poynting-Robertson drag, scattering as they cross the orbit of the planet and naturally creating a dust-depleted region inside its orbit. However, as shown in the upcoming study by Hillenbrand et al. (2007), all our disks are collision-dominated, as are bright debris disks in general (Wyatt 2005). This means that the dust particles may not have time to drift too far from the parent bodies before getting eroded by collisions down to the blow-out size. Thus, in these collision-dominated disks the dust generally traces the location of the planetesimals. Therefore, we need to evaluate the effect of a giant planet on the planetesimal population rather than on the dust particles. Because gravitational scattering is a process independent of mass, the models of Moro-Martín \& Malhotra (2005) are also applicable to planetesimals as long as these can be considered to be "test particles" (i.e., their masses are negligible with respect to that of the planet).

Using the above models, we evaluate what is the mass of the least massive planet that can open a gap in the planetesimal distribution. In order to provide a good model for the evacuated inner gaps we require that the planet scatters out at least $90 \%$ of the planetesimals. A dotted line in Figure 1 shows these lower masses as a function of orbital radius. We find that for planets in the 5-30 AU range, a planet mass of at least 2-5 $M_{\mathrm{J}}$ masses is required. Thus, it is conceivable that giant planets in the mass range $2-5 M_{\mathrm{J}}$ clear the gaps and still remain undetected by our survey. However, we point out that in the cases of most of our targets, and in particular for HD 35850, the parameter space such a planet can occupy is limited.

Given that our high-contrast imaging survey did not find single, large-separation giant planets that may be responsible for clearing the inner disks, we briefly explore alternative mechanisms. Besla $\& \mathrm{Wu}$ (2007) provides a useful summary of the proposed models, and we will only highlight here a particularly interesting proposal proposed recently by Moro-Martin et al. (2007b) for the system HD 38529, where two close-in planets trigger secular resonances that affect the planetesimal population in the outer disk. In this model the eccentricity of the planetesimals at the location of the secular resonances is excited, thus enhancing the rate of collisions and truncating the planetesimal disk.

Given the range of possible mechanisms that may lead to the formation of dust rings, it is probable that in-depth studies of the individual systems will be required for judging the feasibility of the proposed models on a case-by-case basis. However, our nondetections show that the presence of evacuated large inner holes in cold disks could be related to localized dust production or concentration of dust grains by dust-gas interactions rather than dynamical clearing by single massive giant planets.

\section{CONCLUSIONS}

We present results from a high-contrast angular differential imaging survey of eight cold debris disks, selected to have significantly or totally evacuated inner disks. Our observations searched for massive giant planets that may be responsible for carving out the inner holes in the observed cold debris disks. For most of our targets we reach typical sensitivities of 3-7 $M_{\mathrm{J}}$ between 20 and 50 AU separations, but did not identify any likely planet or brown dwarf candidates.

By comparing the derived planet mass upper limits to lower limits derived from dynamical scattering models (typically 2$5 M_{\mathrm{J}}$ between 10 and $30 \mathrm{AU}$ ), we limit the parameter space available for any single planet capable of efficiently clearing out the inner planetesimal disks.

Our survey complements recent direct imaging surveys of nearby young stars, indicating that massive giant planets at large separations are very rare. Cool debris disks with large inner evacuated cavities remained promising possible exceptions to this rule until now. However, the combination of our observational upper limits and theoretical lower limits strongly suggest that massive giant planets at large separations are not present in most of these systems, reinforcing the finding that the outer cutoff for the giant planet distribution is probably at $15 \mathrm{AU}$ or at even smaller semimajor axes (Kasper et al. 2007).

We thank the staff at the Paranal Observatories for the support of the service mode observations. In particular, we are grateful to S. Mengel and G. Lowell-Tacconi for their help with the preparation of the observations. This material is partly based on work supported by the National Aeronautics and Space Administration through the NASA Astrobiology Institute under Cooperative Agreement CAN-02-OSS-02 issued through the Office of Space Science to the Life and Planets Astrobiology Center (LAPLACE). We would like to thank members of the FEPS team for their help in characterizing the target stars and their disks. FEPS is pleased to acknowledge support through NASA contracts 1224768 , 1224634, and 1224566 administered through JPL.

Facilities: VLT(NACO).

\section{REFERENCES}

Ardila, D. R., et al. 2004, ApJ, 617, L147

Baraffe, I., Chabrier, G., Barman, T. S., Allard, F., \& Hauschildt, P. H. 2003, A\&A, 402, 701

Barnes, S. A. 2007, ApJ, 669, 1167

Beichman, C. A., Bryden, G., Rieke, G. H., et al. 2005, ApJ, 622, 1160

Besla, G., \& Wu, Y. 2007, ApJ, 655, 528

Biller, B. A., et al., ApJS, 173, 143

Burrows, A., Sudarsky, D., \& Lunine, J. I. 2003, ApJ, 596, 587

Greaves, J. S., et al. 2005, ApJ, 619, L187

Hillenbrand, L. A.., et al. 2007, ApJ, submitted.

Hollenbach, D., et al. 2005, ApJ, 631, 1180

Janson, M., et al. 2007, AJ, 133, 2442

Kasper, M., Apai, D., Janson, M., \& Brandner, W. 2007, A\&A, 472, 321

Kellner, S. A. 2005, Ph.D. thesis, Univ. Heidelberg

Kim, J. S., et al. 2005, ApJ, 632, 659

Lafreniere, D., et al. 2007, preprint (arXiv: 0705.4290)

Lenzen, R., Close, L., Brandner, W., Biller, B., \& Hartung, M. 2004, Proc. SPIE, 5492, 970

Lenzen, R., et al. 2003, Proc. SPIE, 4841, 944
Levison, H. F., Morbidelli, A., Gomes, R., \& Backman, D. 2007, in Protostars and Planets V, ed. B. Reipurth, D. Jewitt, \& K. Keil (Tucson: Univ. Arizona), 669 Luhman, K. L., Stauffer, J. R., \& Mamajek, E. E. 2005, ApJ, 628, L69

Mamajek, E. E., et al. 2004, ApJ, 612, 496

Marley, M. S., Fortney, J. J., Hubickyj, O., Bodenheimer, P., \& Lissauer, J. J. 2007, ApJ, 655, 541

Marois, C., Lafrenière, D., Doyon, R., Macintosh, B., \& Nadeau, D. 2006, ApJ, 641,556

Masciadri, E., Mundt, R., Henning, T., Alvarez, C., \& Barrado y Navascués, D. 2005, ApJ, 625, 1004

Meyer, M. R., Backman, D. E., Weinberger, A. J., \& Wyatt, M. C. 2007, in Protostars and Planets V, ed. B. Reipurth, D. Jewitt, \& K. Keil (Tucson: Univ. Arizona), 573

Meyer, M. R., et al. 2004, ApJS, 154, 422

2006, PASP, 118, 1690

Morbidelli, A., Levison, H. F., \& Gomes, R. 2007, preprint (astro-ph/0703558)

Moro-Martín, A., \& Malhotra, R. 2005, ApJ, 633, 1150

Moro-Martín, A., et al. 2007a, ApJ, 658, 1312 2007b, ApJ, 668, 1165 
Mueller, M., \& Weigelt, G. 1987, A\&A, 175, 312

Pascucci, I., et al. 2006, ApJ, 651, 1177

Quillen, A. C. 2006, MNRAS, 372, L14

Rousset, G., et al. 2003, Proc. SPIE, 4839, 140

Setiawan, J., et al. 2007, ApJ, 660, L145

Song, I., Zuckerman, B., \& Bessell, M. S. 2003, ApJ, 599, 342
Takeuchi, T., \& Artymowicz, P. 2001, ApJ, 557, 990

Williams, J. P., et al. 2004, ApJ, 604, 414

Wilner, D. J., Holman, M. J., Kuchner, M. J., \& Ho, P. T. P. 2002, ApJ, 569, L115

Wyatt, M. C. 2005, A\&A, 433, 1007

Zuckerman, B., Song, I., \& Bessell, M. S. 2004, ApJ, 613, L65 